Research Paper

\title{
Sensory Nerves Affect Bone Regeneration in Rabbit Mandibular Distraction Osteogenesis
}

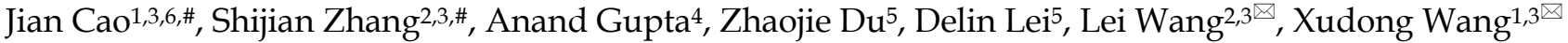 \\ 1. Department of Oral and Craniomaxillofacial Surgery, Shanghai Ninth People's Hospital, College of Stomatology, Shanghai JiaoTong University School of \\ Medicine, Shanghai, China \\ 2. Department of Oral and Maxillofacial-Head \& Neck Oncology, Shanghai Ninth People's Hospital, College of Stomatology, Shanghai JiaoTong University \\ School of Medicine, Shanghai, China \\ 3. National Clinical Research Center for Oral Diseases; Shanghai Key Laboratory of Stomatology \& Shanghai Research Institute of Stomatology, Shanghai, \\ China \\ 4. Government Medical College Hospital, Chandigarh, India \\ 5. Department of Oral and Maxillofacial Surgery, Fourth Military Medical University, School of Stomatology, Xi'an, China \\ 6. Department of Oral and Maxillofacial Surgery, the General Hospital of Lanzhou Command, Lanzhou, China \\ \#, contributed equally to the article.
}

$\square$ Corresponding authors: Dr. Xudong Wang, Department of Oral and Craniomaxillofacial Surgery, Shanghai Ninth People's Hospital, College of Stomatology, Shanghai JiaoTong University School of Medicine; National Clinical Research Center for Oral Diseases; Shanghai Key Laboratory of Stomatology \& Shanghai Research Institute of Stomatology; Shanghai, China; Tel: +86 21 53315159; Email: xudongwang70@hotmail.com and Dr. Lei Wang, Department of Oral and Maxillofacial-Head \& Neck Oncology, Shanghai Ninth People's Hospital, College of Stomatology, Shanghai JiaoTong University School of Medicine; National Clinical Research Center for Oral Diseases; Shanghai Key Laboratory of Stomatology \& Shanghai Research Institute of Stomatology; Shanghai, China; Tel: +86 15921941601; Email: wangleizyh@aliyun.com

(C) Ivyspring International Publisher. This is an open access article distributed under the terms of the Creative Commons Attribution (CC BY-NC) license (https://creativecommons.org/licenses/by-nc/4.0/). See http://ivyspring.com/terms for full terms and conditions.

Received: 2018.11.29; Accepted: 2019.04.11; Published: 2019.06.02

\begin{abstract}
Objectives: To investigate the effects of inferior alveolar nerve on new bone formation in rabbit mandibular distraction osteogenesis.

Methods: 20 New Zealand White rabbits underwent bilateral distraction osteogenesis with a rate of $1 \mathrm{~mm} / \mathrm{day}$. The inferior alveolar nerve of one side was resected under the surgical microscope, with the inferior alveolar vascular intact. The contralateral side received sham operation. The rabbits were sacrificed at consolidation time of 28 days. The regenerate callus underwent radiograph examination, dual-energy X-ray absorptiometry, haematoxylin and eosin staining and histomorphometric analysis. A paired t-test was performed using SPSS 16.0 software package.

Results: The BMD of the new bone in the distraction gap on the denervation side of mandibular was significantly lower $(\mathrm{P}<0.05)$ than on the control side. The histological investigation showed that the bone trabeculae were dis-arrayed containing dispersed cartilage cells on the denervation side, whereas the bone trabeculae were orderly with rich blood vessels and no cartilage cell on the control side. Both new bone volume and the thickness of new trabeculae were significantly lower on the denervation side than on the control side $(P<0.05)$.

Conclusion: The loss of the sensory nerves could result in a decrease of the new bone quality during the mandibular distraction osteogenesis.
\end{abstract}

Key words: Distraction osteogenesis; Sensory nerves; Denervation; Bone regeneration

\section{Introduction}

The peripheral nervous system is critically involved in bone metabolism, osteogenic differentiation of precursor cells, bone mineralization, and bone remodeling ${ }^{1}$. It was reported that in patients with spinal cord injuries, a profound decrease of bone mineral density was measured in comparison with controls ${ }^{2}$. Experimental studies have also provided accumulating evidences that peripheral nerve fibers not only are important in normal bone homeostasis and skeletal growth ${ }^{3}$, but also have influence on repair 
mechanism of bone fracture ${ }^{4}$. Peripheral nerve fibers, including sympathetic and sensory nerves, frequently innervate trabecular bone, periosteum, and fracture callus. Among them, the sensory nerves in general contain two different nociceptive neuropeptide families: the tachykinins and calcitonin gene-related peptides, which suppress bone resorptive activities through regulation of RANKL/OPG expression ${ }^{1,4}$. However, it remains unclear whether the peripheral nerve ingrowth is crucial to the new bone formation in the scenario of endogenous or exogenous bone regeneration, such as distraction osteogenesis and bone tissue engineering respectively.

Distraction osteogenesis (DO) has been widely applied in the treatment of bone defects and deformities in orthopedics and craniomaxillofacial surgery ${ }^{5}$. During the process of DO, the osteotomy followed by gradual distraction yields two vascularized bone surfaces and induces new bone formation, and the intramembranous bone formation is the predominant mechanism of ossification in which neo-callus formation occurs through the direct differentiation of mesenchymal stem cells into osteoblast lineages $^{6}$. Therefore, as a thoroughly new way of bone regeneration instead of wound repairing, DO provides an ideal research model for us to better understand the mechanism of the initiation, development and ossification of new bone formation.

In our previous study, we have demonstrated that the injection of Nerve Growth Factor $\beta$ at the end of the distraction period significantly enhanced new bone formation in a rabbit model of mandibular distraction osteogenesis ${ }^{7}$. In the present study, we hypothesize that the denervation of the mandible could result in a decrease of new bone formation in the distraction osteogenesis. We use the rabbit model of bilateral mandibular distraction osteogenesis with the inferior alveolar nerve resected on one side to determine the effects of sensory nerve on the new bone formation. We show that the new bone quality was significantly lower on the denervation side than on the control side at the end of consolidation time of 28 days.

\section{Material and Methods}

\section{Animal model of bilateral mandibular distraction osteogenesis}

Twenty skeletally mature (2.8-3.2 kg), male, New Zealand White rabbits were included. The animals were housed and cared for in accordance with the guidelines established by the Animal Center for Medical Experiment at Fourth Military Medical University. All the animal protocols were approved by the Animal Care and Use Committee at the Fourth
Military Medical University (approval number: 15DW0933). The details of the model of bilateral mandibular distraction osteogenesis in rabbits were described previously ${ }^{8}$. Briefly, the animals were anaesthetized with $1.0 \%$ pentobarbital sodium 30 $\mathrm{mg} / \mathrm{kg}$ injected intra-peritoneum. After exposure of the bilateral mandibular body and ramus through the bilateral submandibular incision, a titanium distractor (Zhongbang Titanium Biomaterials Corporation, $X i^{\prime}$ an, China) was fixed along the buccal surface of the mandible, with the distraction rod emerging into the labial vestibule. Then the vertical osteotomies were performed bilaterally between the premolar teeth and mental foramen using a fissure bur, with the care of avoiding injury to the inferior alveolar nerve (IAN) (Fig. 1).

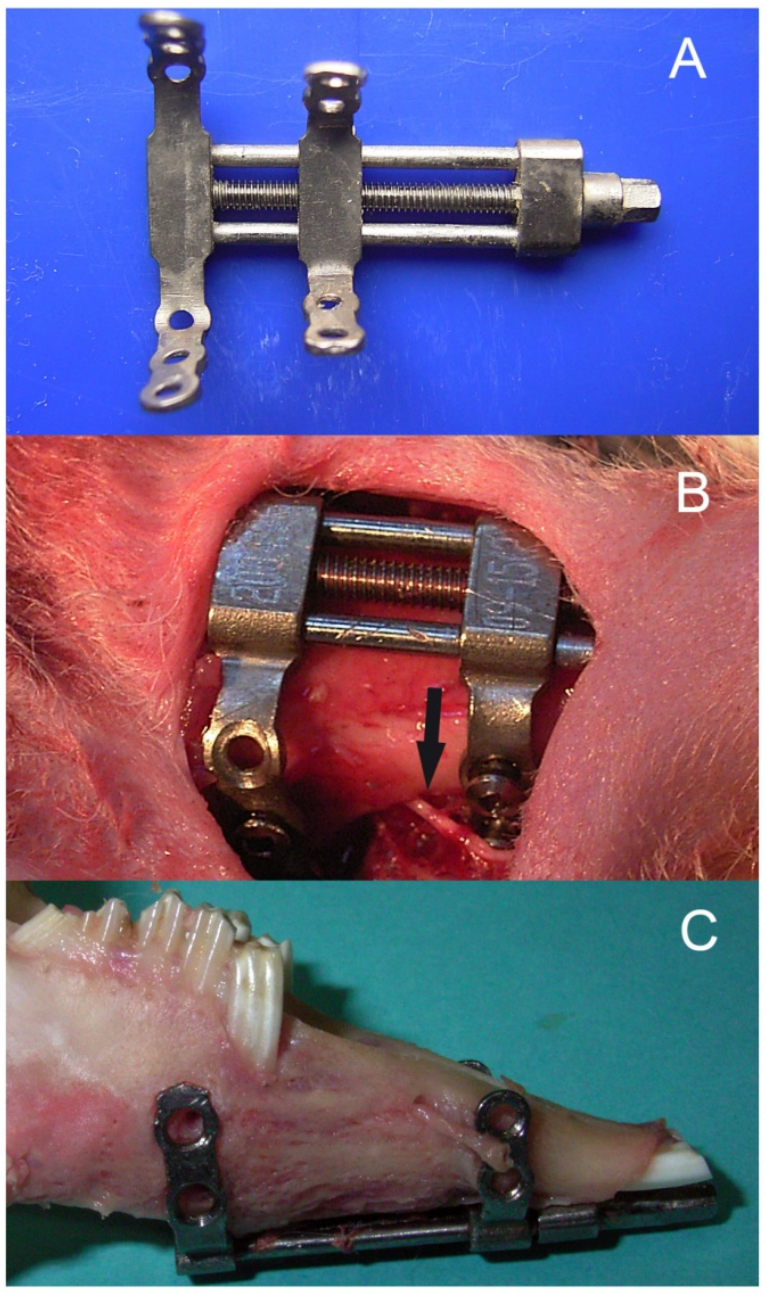

Fig. 1. The bilateral mandibular distraction osteogenesis in rabbits: (A) bilateral distractor, (B) a photograph during surgery, and (C) the elongated mandiblular. Note the inferior alveolar nerve.

\section{Denervation operation and distraction protocol}

After the bilateral osteotomies performed completely, the left mandibular (experimental side) 
was denervated and the right mandibular was the control side according to the following procedure. Firstly, we removed part of the cortical bone on the mandibular margin ahead of the antegonial notch and exposed the inferior alveolar neurovascular bundle. Then we isolated the IAN from the vascular carefully under the surgical microscope. A length of $6 \mathrm{~mm}$ IAN was resected and ligated on the both cross sections with the vascular intact (Fig. 2). A sham operation was performed on the right mandibular of the same animal to create a control, which included the exposure of the inferior alveolar neurovascular bundle and keep the IAN intact. After a latency period of 5 days, the gradual distraction was performed at a rate of $0.5 \mathrm{~mm}$ per 12 hours for 10 days. Then the regenerated bone was allowed to consolidate for an additional 28 days. During this period, animals were fed with semifluid food. Animals were sacrificed with an overdose of pentobarbital sodium, and the callus from the distraction gap was harvested, demineralized, and prepared for staining with haematoxylin and eosin $(\mathrm{H}$ and E).

\section{Sample harvesting and radiographic examination}

At consolidation time of 28 days, all the rabbits were sacrificed. After the carotid artery perfusion, both sides of the mandible were harvested with the soft tissue excised. The internal fixation of the tissue was performed using $2.0 \%$ paraformaldehyde and $2.5 \%$ glutaraldehyde in $0.1 \mathrm{M} / \mathrm{L}$ phosphate-buffered saline ( $\mathrm{pH}$ 7.4). Plain radiographs were taken and the mandibles were scanned under a dual-energy X-ray absorptiometry (DEXA, Lunar DPX-1Q, Lunar Radiation Corporation, Madison, WI) to examine their bone mineral density (BMD).

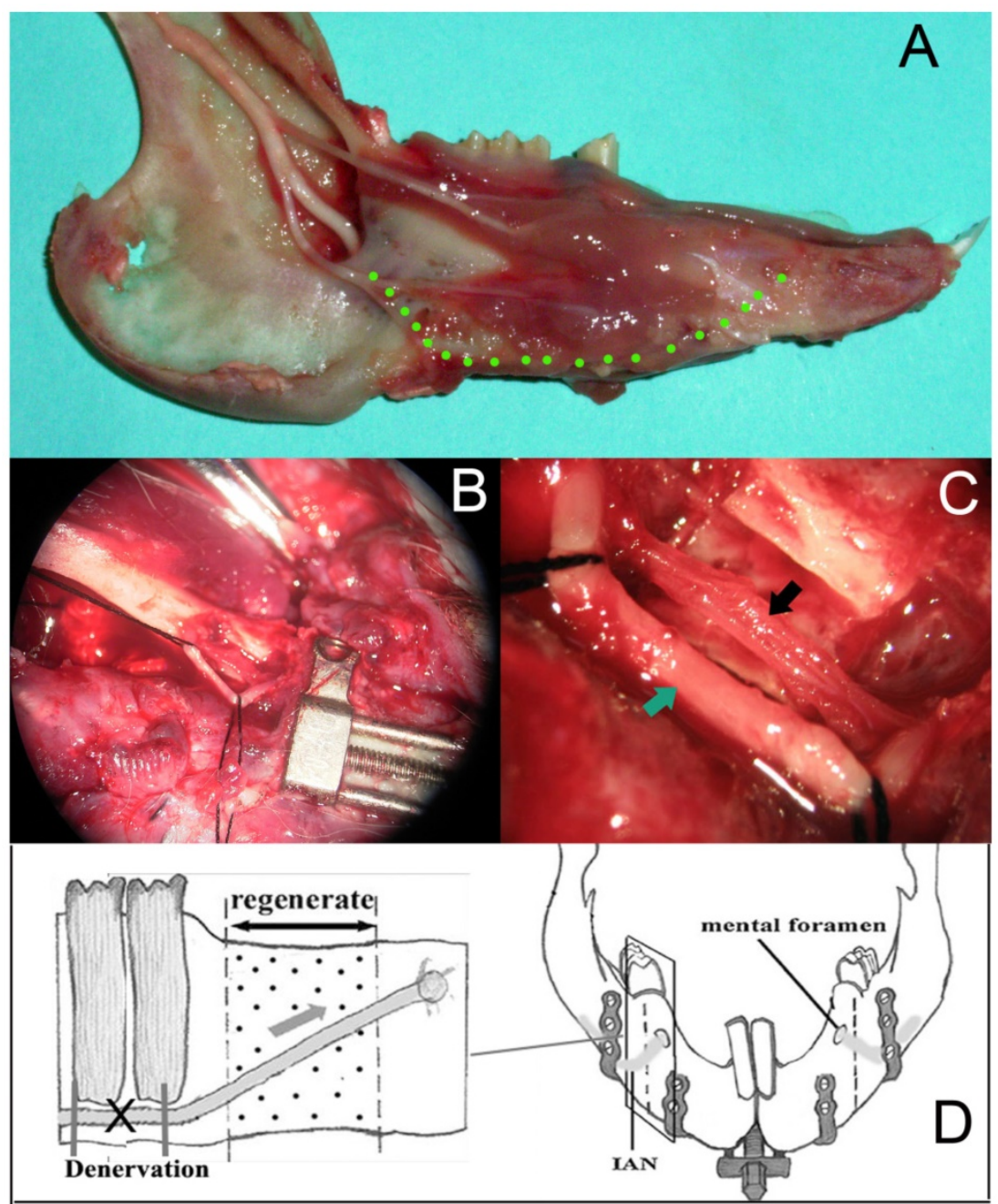

Fig. 2. (A) The pathway of the IAN in the rabbit mandibular; (B)after the removal of the cortical bone on the mandibular margin ahead of the antegonial notch, the IAN was exposed under the surgical microscope (4×); (C)a length of $6 \mathrm{~mm}$ IAN was resected and ligated on the both cross sections with the vascular intact under the surgical microscope (16×); (D) a scheme for the procedure of denervation. 


\section{Bone histology and histomorphometry}

After the radiographic examination, the mandibles were cut into specimens including $2 \mathrm{~mm}$ of neighboring normal bone. The specimens were decalcified in buffered 14.5\% EDTA ( $\mathrm{pH} 7.3$ ) for 20 to 30 days, then dehydrated, and paraffin embedded. Each block was cut into 5 - $\mu \mathrm{m}$-thick sections in the axial plane and stained with hematoxylin and eosin. Bone histomorphometric analysis was performed on 4 sections for each sample using National Institutes of Health (NIH) Image analysis (ImageJ v1.51). Eight fields were randomly selected from each section and measured twice with a 3-day interval by a single, unbiased examiner who was blinded to the experimental groups. Bone volume/total volume (BV/TV, \%, ratio of bone volume to the total tissue volume of distracted region) and trabecular thickness (Tb. Th, $\mu \mathrm{m}$ ) were analyzed as the bone histomorphometric parameters. In each field, we selected the overall pixels of the area of the new bone. The ratio of the bone volume to total tissue volume was then calculated. As for Tb.Th, we first marked the margin of the trabecular bone in each section. Then local thickness is defined for every point inside the trabecular bone as the diameter of largest inscribed sphere centered at any point in the medial axis.
Finally, global thickness is estimated as the mean of the local thickness at every point inside the object.

\section{Statistical analysis}

All data were presented as the mean and standard deviation of the mean. Bone densitometry and histomorphometric results were statistically analyzed by a paired t-test for comparisons between 2 groups. Statistical analysis was performed using SPSS 16.0 software. A value of $\mathrm{P}<0.05$ was interpreted to denote statistical significance.

\section{Results}

All 20 rabbits tolerated the experimental procedure well, with weight loss of less than $10 \%$. In all of the animals, lengthening of $9.11 \pm 0.69 \mathrm{~mm}$ was successfully achieved and bone consolidation was obtained by the end of the experiment. We have measured the length, height and width of the regenerated bones using a caliper, and no difference $(\mathrm{P}>0.05)$ in regenerated bone dimensions was observed between the both sides of mandibles (data not shown). No nonunion was observed in all the specimens. The mental nerve on the experimental side atrophied significantly while the counterpart on the control side showed quite normal.

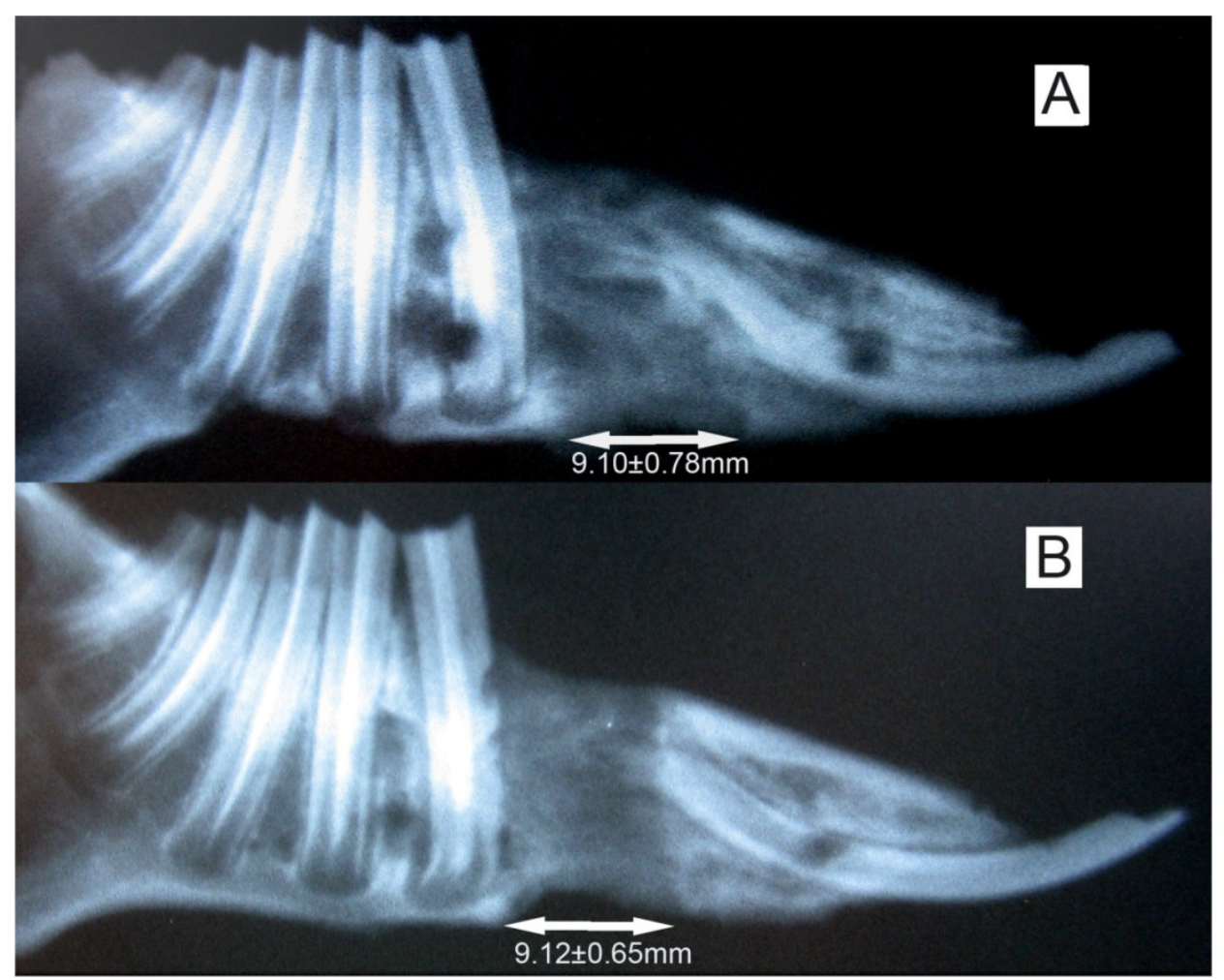

Fig. 3. The $\mathrm{X}$-ray of the elongated mandibular on the consolidation time of 28 days. ( $\mathrm{A}$ ) the denervation side; (B) the control side. The arrows denote the elongated bone tissue. Lengthening of $9.11 \pm 0.69 \mathrm{~mm}$ was successfully achieved, and no difference $(P>0.05)$ in regenerated bone dimensions was observed between the both sides of mandibles. 


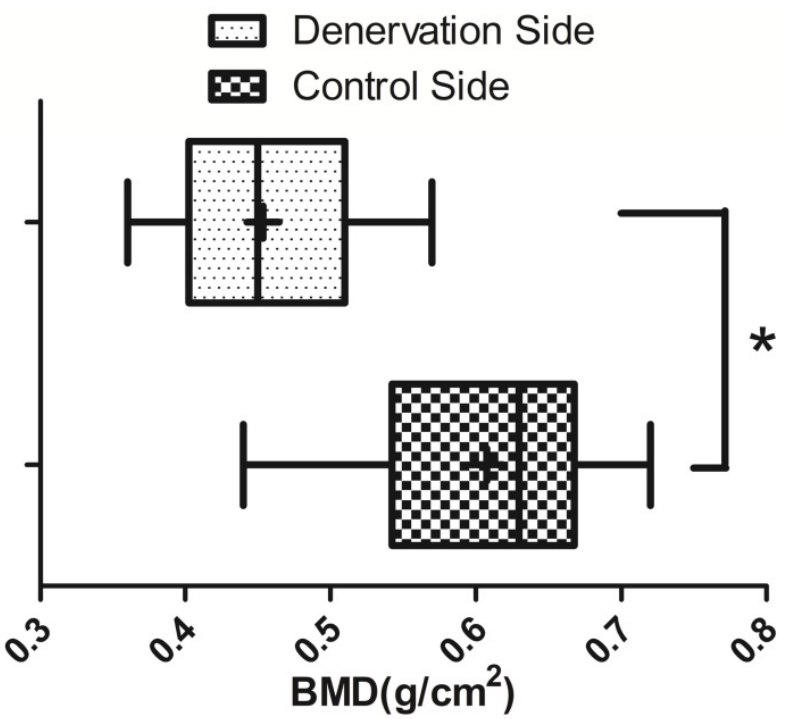

Fig. 4. $B M D(\bar{x} \pm s)$ of the new bone in the distraction gap on the experimental side and the control side $(\mathrm{N}=20) . * P<0.05$.

\section{Radiographic examination and BMD}

Radiographs showed that the distraction gap was filled with new bone in all of the animals. There was a lower density of the new bone on the experimental side than on the control side (Fig. 3).
BMD of the new bone in the distraction gap on the experimental side was $0.46 \pm 0.07 \mathrm{~g} / \mathrm{cm}^{2}$, which is significantly lower $(P<0.05)$ than on the control side $\left(0.61 \pm 0.09 \mathrm{~g} / \mathrm{cm}^{2}\right)$ (Fig. 4$)$.

\section{Bone histologic and histomorphometric analysis}

At 4 weeks post lengthening, the distraction gaps of all the animals were completely united with bone tissue. On the experimental side of mandible, the bony trabeculae had various degrees of consolidation with occasional fibrous and cartilaginous tissues, and there was initial replacement of woven bone by lamellar bone. On the control side of mandible, distraction gaps mainly consisted of well-organized woven bone and lamellar bone, formed in parallel to the distraction forces, with signs of callus remodeling and no fibrous or cartilaginous tissues (Fig. 5). In comparison, bone consolidation and remodeling were more advanced on the control side. At the 28 days of consolidation time, bone volume/total volume $(\mathrm{BV} / \mathrm{TV})$ and thickness of new trabeculae (TNT) on the experimental side were significantly lower $(P<0.05)$ than on the control side (Fig. 6).
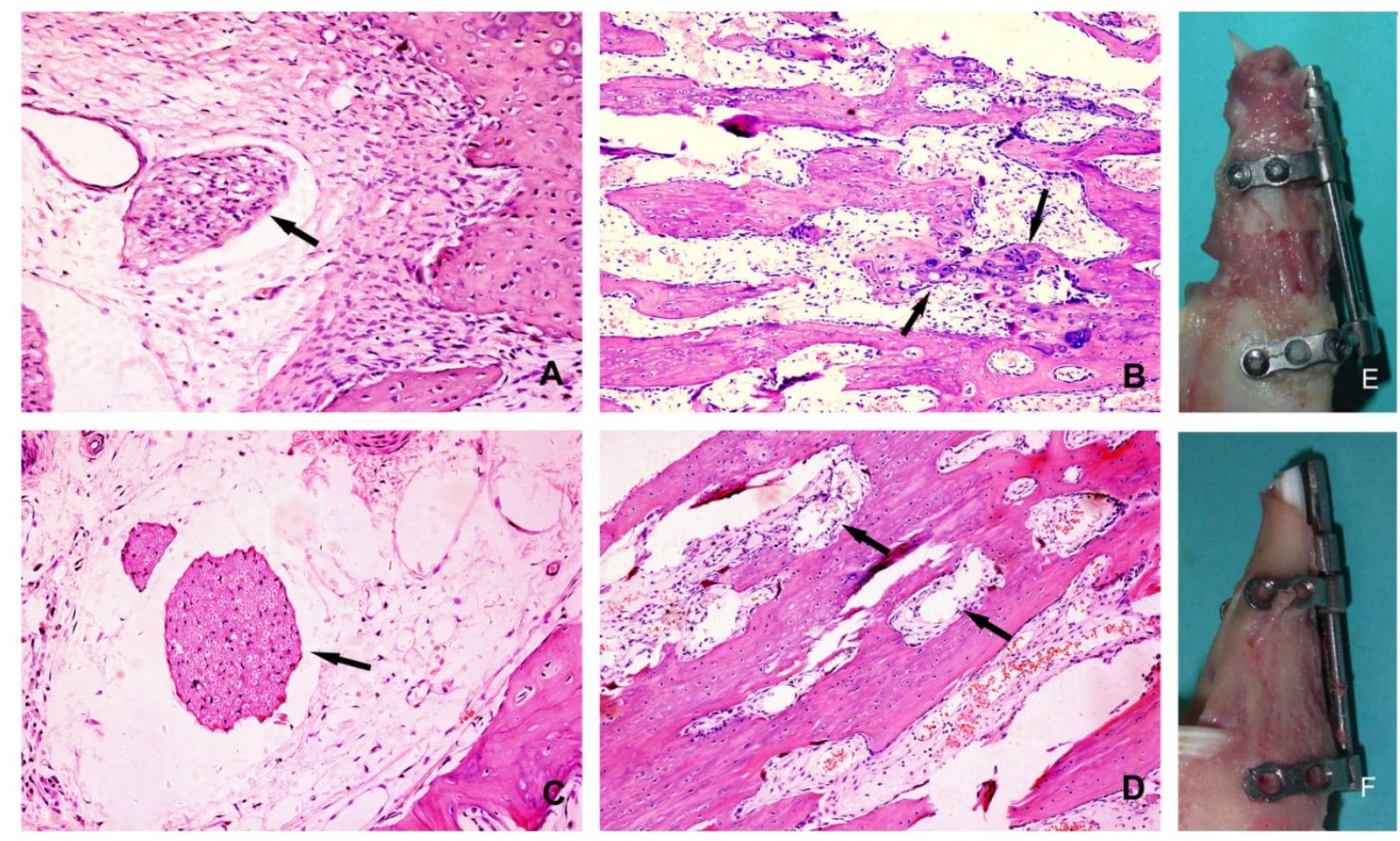

Fig. 5. Histological section of the regenerated bone after 28 days of consolidation: $(A)$ the experimental side(200x): the arrow denotes the degenerated nerve tissue; (B) the experimental side $(100 \times)$ : the bone trabeculae disarrayed, the arrows denote the dispersed cartilage cells; (C) the control side(200x): the arrow denotes the undamaged nerve tissue; (D) the control side $(100 \times)$ : the trabeculae were oriented along the direction of force, the arrows denote rich blood vessels; (E) The overview of the callus on the experimental side; (F) The overview of the callus on the control side. 
Denervation Side

$\mathbf{D}$ Control side
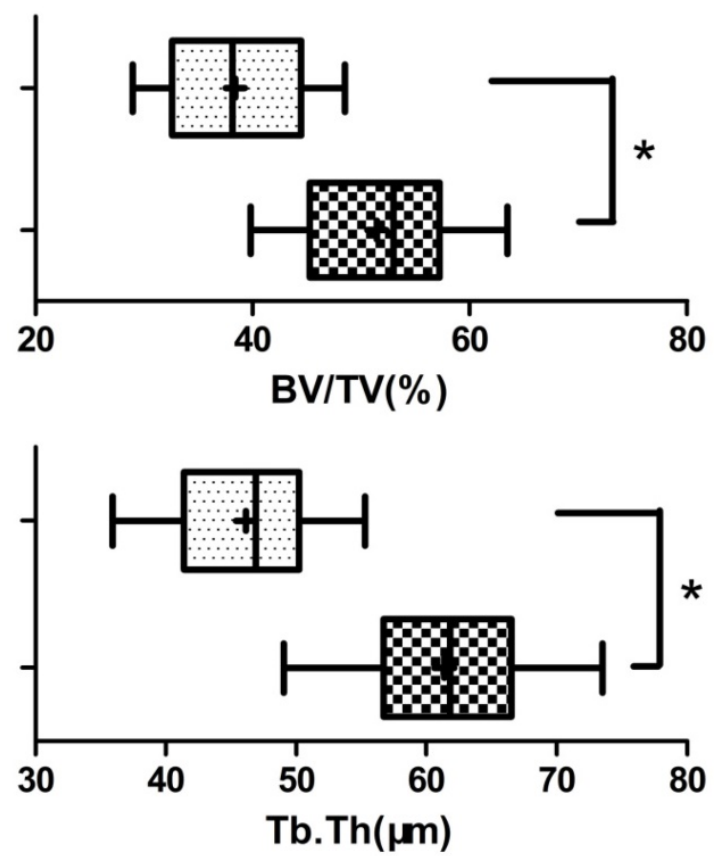

Fig. 6. Bone histomorphometric analysis $(\bar{x} \pm s)$ of the new bone at the 28 days of consolidation time. The bone volume/total volume (BV/TV) and thickness of new trabeculae (Tb.Th) on the experimental side were significantly lower than on the control side $(\mathrm{N}=20)$. $* P<0.05$

\section{Discussion}

Several researches have demonstrated an intensive network of peripheral nerve fibers within the skeleton, not only in the periosteum but also within trabecular, cortical bone, bone marrow, and epiphyseal growth plate, ${ }^{9,10}$. Many of those nerve fibers, including sensory and sympathetic nerves, are associated with blood vessels, but several blood vessel-unrelated nerves and free nerve endings have also been observed. Besides the possibility that sensory and sympathetic nerve fibers have important roles in skeletal pain transmission, accumulating evidence suggests that sensory and sympathetic nerve fibers do have a role in bone remodeling and osteogenic differentiation of precursor cells during skeletal growth. In bone, the areas with the highest metabolic activity receive the richest sensory and sympathetic innervation ${ }^{11}$. Furthermore, bone cells express receptors for many of the neuronal messengers present in these skeletal nerve fibers, and activation of such receptors leads to profound effects on the activity of both osteoblasts and osteoclasts, strongly suggesting the existence of neuro-osteogenic or neuro-immuno-osteogenic interactions ${ }^{12}$. During endochondral ossification, sensory neuropeptide SP promotes proliferation of stem cells and growth plate chondrocytes, whereas signaling through $\beta$-ARs inhibits chondrogenic differentiation of osteo-chondroprogenitor cells and terminal differentiation of chondrocytes. In bone metabolism and bone remodeling, CGRP and VIP have anabolic effects, inducing osteoblast activity and inhibiting osteoclastogenesis, whereas SP also has catabolic effects depending on its concentration ${ }^{9}$. Our previous study also demonstrated that the injections of hNGF $\beta$ to the regenerate zone following the end of distraction could significantly increase myelinated fiber density of the IAN, as well as enhance bone consolidation in a rabbit model of mandibular $\mathrm{DO}^{13}$. Therefore, it is reasonable to assume that the peripheral sensory nervous system plays an important role in bone regeneration.

The repairing of bone defects and promoting of bone regeneration is still a major issue that has not been well resolved to date. Especially for the defects beyond the critical size, autologous or allogeneic bone graft is often a have-to-do option. Distraction osteogenesis is a unique postnatal new bone formation process based on the "tension-stress principle", as proposed by Ilizarov ${ }^{14}$. The basic premise is that new bone formation is induced by the gradual distraction of the fracture callus after a low-energy corticotomy with careful preservation of the soft tissue envelope surrounding the bone. Neovascularization is critically required for successful bone formation in this process, and systemic factors associated with neovascularization also affect the bone regeneration ${ }^{15}$. In the present study, we demonstrate that the denervation of the distraction gap lead to a decrease of the new bone quality, which corroborates the study by Offley and colleagues, who used selective lesioning of the unmyelinated sensory neural pathway to determine the role of capsaicin-sensitive sensory SP- and CGRP-containing afferents in the maintenance of normal bone balance in skeletally mature rats. Collectively, their results indicate that capsaicin-sensitive sensory neurons contribute to skeletal homeostasis and that lesioning these neurons caused enhanced bone resorption, a reduction in new bone formation, a subsequent loss of trabecular connectivity and thickness, and ultimately an increase in bone fragility ${ }^{16}$.

The rabbit model of mandible lengthening was well established and had been used extensively to perform callus stimulation studies ${ }^{17}$. A relatively safe rate of bone lengthening was recommended to be about $1.0 \mathrm{~mm} / \mathrm{d}$ in several animal models of mandibular DO, and rates faster than $2 \mathrm{~mm} / \mathrm{d}$ could result in a poor quality of bone formation ${ }^{18}$. To investigate the effects of the denervation on distraction osteogenesis, we adopted a new method of inferior alveolar nerve transection ${ }^{19}$. We exposed the 
inferior alveolar neurovascular bundle, isolated the IAN from the vascular carefully under the surgical microscope, then resected a length of $6 \mathrm{~mm}$ IAN and ligated the both cross sections of the IAN in case of the reunion of the nerve ends. Although it is possible that a very small amount of peripheral nerve fibers may grow into the callus through the periosteum during the distraction period, this denervation procedure provides an ideal model for investigating the interplay between sensory nerves and the bone regeneration.

In conclusion, we have demonstrated that the denervation of the mandibular could result in a decrease of the new bone quality in distraction osteogenesis. Although future improvements of the study should include the immunohistochemistry and confocal microscopy to examine the budding, sprouting and ingrowth of the peripheral nerve fibers in the new bone, it suggested that the sensory nerves play an important role in the new bone formation during the mandibular distraction osteogenesis.

\section{Acknowledgements}

This work was supported by National Natural Science Foundation of China (No. 81771046, 81400552 and 81270015), programs of Shanghai Talent Development (No. 2018042) and Shanghai Summit \& Plateau Disciplines.

\section{Competing Interests}

The authors have declared that no competing interest exists.

\section{References}

1. Grässel S. The role of peripheral nerve fibers and their neurotransmitters in cartilage and bone physiology and pathophysiology. Arthritis Res Ther. 2014;16(6):485.

2. Dauty M, Perrouin Verbe B, Maugars Y, Dubois C, Mathe JF. Supralesional and sublesional bone mineral density in spinal cord-injured patients. Bone. 2000;27(2):305-309.

3. Niedermair T, Kuhn V, Doranehgard F, et al. Absence of substance P and the sympathetic nervous system impact on bone structure and chondrocyte differentiation in an adult model of endochondral ossification. Matrix Biol. 2014;38:22-35.

4. Li J, Kreicbergs A, Bergström J, Stark A, Ahmed M. Site-specific CGRP innervation coincides with bone formation during fracture healing and modeling: A study in rat angulated tibia. J Orthop Res. 2007;25(9):1204-1212.

5. McCarthy JG, Stelnicki EJ, Mehrara BJ, Longaker MT. Distraction osteogenesis of the craniofacial skeleton. Plast Reconstr Surg. 2001;107(7):1812-1827.

6. Al-Aql ZS, Alagl AS, Graves DT, Gerstenfeld LC, Einhorn TA. Molecular mechanisms controlling bone formation during fracture healing and distraction osteogenesis. J Dent Res. 2008;87(2):107-118.

7. Cao J, Wang L, Lei D, Liu Y-P, Du Z, Cui F-Z. Local injection of nerve growth factor via a hydrogel enhances bone formation during mandibular distraction osteogenesis. Oral Surg Oral Med Oral Pathol Oral Radiol. 2012;113(1):48-53.

8. Wang L, Cao J, Lei D, et al. Effects of nerve growth factor delivery via a gel to inferior alveolar nerve in mandibular distraction osteogenesis. J Craniofac Surg. 2009;20(6):2188-2192.

9. Lerner UH, Persson E. Osteotropic effects by the neuropeptides calcitonin gene-related peptide, substance $\mathrm{P}$ and vasoactive intestinal peptide. J Musculoskelet Neuronal Interact. 2008;8(2):154-165.

10. Hukkanen M, Konttinen YT, Santavirta S, et al. Rapid proliferation of calcitonin gene-related peptide-immunoreactive nerves during healing of rat tibial fracture suggests neural involvement in bone growth and remodelling. Neuroscience. 1993;54(4):969-979.
11. Chartier SR, Mitchell SAT, Majuta LA, Mantyh PW. The Changing Sensory and Sympathetic Innervation of the Young, Adult and Aging Mouse Femur. Neuroscience. 2018;387:178-190.

12. Tomlinson RE, Li Z, Zhang $Q$, et al. NGF-TrkA Signaling by Sensory Nerves Coordinates the Vascularization and Ossification of Developing Endochondral Bone. Cell Rep. 2016;16(10):2723-2735.

13. Wang L, Cao J, Lei DL, et al. Application of nerve growth factor by gel increases formation of bone in mandibular distraction osteogenesis in rabbits. Br J Oral Maxillofac Surg. 2010;48(7):515-519.

14. Ilizarov GA. Clinical application of the tension-stress effect for limb lengthening. Clin Orthop Relat Res. 1990;250(250):8-26.

15. Weiss S, Zimmermann G, Baumgart R, Kasten P, Bidlingmaier M, Henle P. Systemic regulation of angiogenesis and matrix degradation in bone regeneration - Distraction osteogenesis compared to rigid fracture healing. Bone. 2005;37(6):781-790.

16. Offley SC, Guo TZ, Wei $\mathrm{T}$, et al. Capsaicin-sensitive sensory neurons contribute to the maintenance of trabecular bone integrity. J Bone Miner Res. 2005;20(2):257-267.

17. Li G, Bouxsein ML, Luppen C, et al. Bone consolidation is enhanced by rhBMP-2 in a rabbit model of distraction osteogenesis. J Orthop Res. 2002;20(4):779-788.

18. Al Ruhaimi KA. Comparison of different distraction rates in the mandible: An experimental investigation. Int J Oral Maxillofac Surg. 2001;30(3):220-227.

19. Yamashiro $T$, Fujiyama $K$, Fujiyoshi $Y$, Inaguma N, Takano-Yamamoto $T$. Inferior alveolar nerve transection inhibits increase in osteoclast appearance during experimental tooth movement. Bone. 2000;26(6):663-669. 